\title{
Role of endogenous glucocorticoids in hyperalgesia and edema in old arthritic rats
}

C.M. Yokoro,

M.A.K.F. Tatsuo,

L.S.M. Pereira,

D.L.F. Alves and

J.N. Francischi
Departamento de Farmacologia, Instituto de Ciências Biológicas, Universidade Federal de Minas Gerais, Belo Horizonte, MG, Brasil

\section{Correspondence}

J.N. Francischi

Departamento de Farmacologia ICB, UFMG

Av. Antônio Carlos, 6627

31270-901 Belo Horizonte, MG

Brasil

Fax: +53-31-3499-2695

E-mail: janettif@mono.icb.ufmg.br

Research support by FAPEMIG (No. 867/97), CNPq and CAPES.

J.N. Francischi, D.L.F. Alves and

C.M. Yokoro are recipients of

research and doctoral fellowships

from $\mathrm{CNPq}$, respectively.

Received February 1, 2002

Accepted September 2, 2002

\section{Abstract}

We compared the intensity and frequency of arthritis in old (8-12 months, $\mathrm{N}=12)$ and juvenile ( 2 months, $\mathrm{N}=10)$ rats and determined the role played by adrenal glands in this disorder. Arthritis was induced by subcutaneous injection of Mycobacterium butyricum at the base of the tail of female Holtzman rats at day zero. Paw edema and hyperalgesia were monitored from day zero to day 21 after induction as signs of arthritis development. Some $(\mathrm{N}=11)$ old animals were adrenalectomized bilaterally and treated with dexamethasone or celecoxib immediately following surgery. All bilaterally adrenalectomized old animals became susceptible to arthritis and the onset of disease was shortened from the 10th to the 5th day. Hyperalgesia and paw edema responses were less frequent in older animals (50 and 25\% compared to control juvenile rats, respectively), although old responder animals showed responses of similar intensity to those of their juvenile counterparts: by the 14th day the data for hyperalgesia were juvenile $=0.8 \pm 0.07 /$ old $=0.8 \pm 0.09$, and for paw edema juvenile $=$ $56.6 \pm 6.04 /$ old $=32.24 \pm 12.7$, reported as $\Delta \%$ increase in paw edema. Chronic treatment of adrenalectomized old animals with dexamethasone $(0.01$ or $0.1 \mathrm{mg} / \mathrm{kg}$ ) but not celecoxib $(3 \mathrm{mg} / \mathrm{kg})$, once daily for 21 days by gavage, abolished the effects of adrenalectomy, in particular those related to the hyperalgesia response (old $=0.95 \pm 0.03$ /dexamethasone $=0 \pm 0 ; 14$ th day), thus suggesting a specific participation of circulating corticosteroids in the modulation of pain in old arthritic rats.

\section{Introduction}

Rheumatoid arthritis is an autoimmune chronic inflammatory disease characterized by infiltration and activation of inflammatory cells within the synovial tissue of multiple joints (1). It is most common in older people, especially women, and is a source of considerable morbidity and mortality in the western world (2). There have been impor-
Key words - Hyperalgesia

- Edema

- Adjuvant arthritis

- Adrenalectomy

- Dexamethasone

- Old rats tant advances in the understanding of the pathophysiology of various types of human arthritis, especially in the case of rheumatoid arthritis. These advances were made possible at least in part by the availability of the adjuvant-induced arthritis model developed in rodents (3-5).

We have used the adjuvant-induced arthritis model to study the efficacy and the mode of action of analgesic, anti-inflammatory and im- 
munosuppressant drugs (6-8). In this model, articular hyperalgesia starts between the 7 th and 10th day of disease induction, whereas paw edema usually appears later, being evident by the 14th day (7). Using this model, we have observed that, in contrast to the human disease, older rats are less susceptible to the disease than juvenile rats. Older mice are also less susceptible to systemic lupus erythematosus, another model of autoimmune disease (9).

Thus, the objective of the present study was to determine the intensity and frequency of hyperalgesia and edema in old and juvenile rats of the same lineage and sex, and to assess the role of adrenal glands in the development of arthritis in old rats, since corticosteroids were first found to have anti-inflammatory activity in arthritic patients (10).

\section{Material and Methods}

\section{Animals}

Juvenile, 2-month-old, or old, 8- to 12month-old, female Holtzman rats weighing 140-170 and 270-300 g, respectively, were used. The animals were kept in cages (maximum of five animals per cage) at $26 \pm 3^{\circ} \mathrm{C}$ and on a 12-h light/dark cycle with free access to standard laboratory rat pellets and water. The ethical guidelines of the International Association for the Study of Pain in conscious animals were followed (11).

\section{Induction of arthritis with an adjuvant}

Rats were injected subcutaneously with a single dose of $0.2 \mathrm{ml}$ mineral oil-water emulsion $(10: 1, \mathrm{v} / \mathrm{v})$ containing $400 \mu \mathrm{g}$ dry Mycobacterium butyricum into the dorsal root of the tail under ether anesthesia. The day of adjuvant injection is referred to as day zero.

\section{Measurement of hind paw hyperalgesia and edema}

The method for measuring hyperalgesia has been described previously (12). Briefly, the tendency of normal (naive), control and arthritic rats to vocalize following flexion of the tarsotibial joints of both hind paws was tested daily for 21 days starting from day 0 . Hyperalgesia is reported as the mean $\pm \mathrm{SEM}$ number of vocalizations following five flexions of the hind limb tarsotibial joints, considering maximal hyperalgesia $($ value $=1$ ) when five vocalizations were obtained following five flexions of the paws. Hind paw volume (as an indicator of edema) was measured daily using an Ugo Basile hydroplethysmometer (model 7150, Comerio, Varese, Italy) following the hyperalgesia test. The volume $(\mathrm{ml})$ of a hind paw was essentially the same as that of the contralateral paw (data not shown) and is reported as the mean \pm SEM in $\mathrm{ml}$. The increase in paw volume is also reported as percent of the control paw. All measurements were made at the same time of day.

An animal was considered to be arthritic when the mean value for hyperalgesia was above 0.3 and to present edema when the difference $(\Delta)$ in paw volume between each day and day zero was $\geq 0.3 \mathrm{ml}$.

\section{Bilateral adrenalectomy}

Complete removal of the adrenal (13) was performed in two groups of old female rats: controls, which were sham-adrenalectomized, and bilaterally adrenalectomized animals. All animals were injected with the adjuvant (day 0) 5 days after adrenalectomy and followed as previously described. Food containers and water and saline $(0.15 \mathrm{M}$ $\mathrm{NaCl}$ ) bottles were attached to the cages and daily intake by the animals was monitored throughout the experiment.

\section{Treatment with anti-inflammatory drugs}

Dexamethasone $(0.1$ or $0.01 \mathrm{mg} / \mathrm{kg})$ or its vehicle $(0.15 \mathrm{M} \mathrm{NaCl})$ was administered by gavage to adrenalectomized adjuvant- 
treated old rats immediately after adrenalectomy and continued through the 21 st day of arthritis induction. Sham-operated animals were treated with $0.15 \mathrm{M} \mathrm{NaCl}$ by the same route and for the same time as adrenalectomized animals. Another group of adrenalectomized adult animals received celecoxib (3 $\mathrm{mg} / \mathrm{kg}$ ) daily by gavage for 21 days after adrenalectomy. This dose of celecoxib is analgesic in the standard model of hyperalgesia induced by carrageenan in rat paws (data not shown).

\section{Drugs and M. butyricum}

The following drugs and the bacterium were purchased from the sources indicated: dexamethasone (Decadron, Prodome, Campinas, SP, Brazil), celecoxib (Celebra, Searle, Caguas, Porto Rico), and M. butyricum (Difco Laboratories, Detroit, MI, USA).

\section{Data analysis}

Results are reported as means \pm SEM and were compared by one-way analysis of variance (ANOVA). Data concerning the mean percent increase of volume were compared using two-way analysis of variance (ANOVA) for multiple comparisons. The software used for statistical analysis was SigmaStat ${ }^{\circledR}$.

\section{Results}

\section{Development of adjuvant arthritis in young and old rats}

The first series of experiments was designed to compare the development of adjuvant-induced arthritis in juvenile and old rats. Hyperalgesia, the increase in vocalization in response to forced flexion of the tarsotibial joints, was evident from the 10th day in juvenile animals (circles), reaching a maximum value by the 21 st day of disease induction (Figure 1A). The results concerning the juvenile control group were omitted from Figure 1A for the sake of clarity since the animals did not develop hyperalgesia or edema. In contrast to juvenile animals, however, only half of the adjuvant-induced old animals, i.e., 6 of 12 animals, presented hyperalgesia, corresponding to a rate of $50 \%$ responder animals (filled squares, upper line in Figure 1B). The hyperalgesia presented by old responder animals (filled squares), however, was of the same magnitude as that presented by juvenile animals (circles). In contrast to the $50 \%$ responder rate for hyperalgesia in old animals, the rate for paw edema was only about $25 \%$ ( 3 of 12 , Figure 1D) compared to $100 \%$ for juvenile rats (Figure $1 C)$. There was greater variability in the edema values of old animals (filled squares).

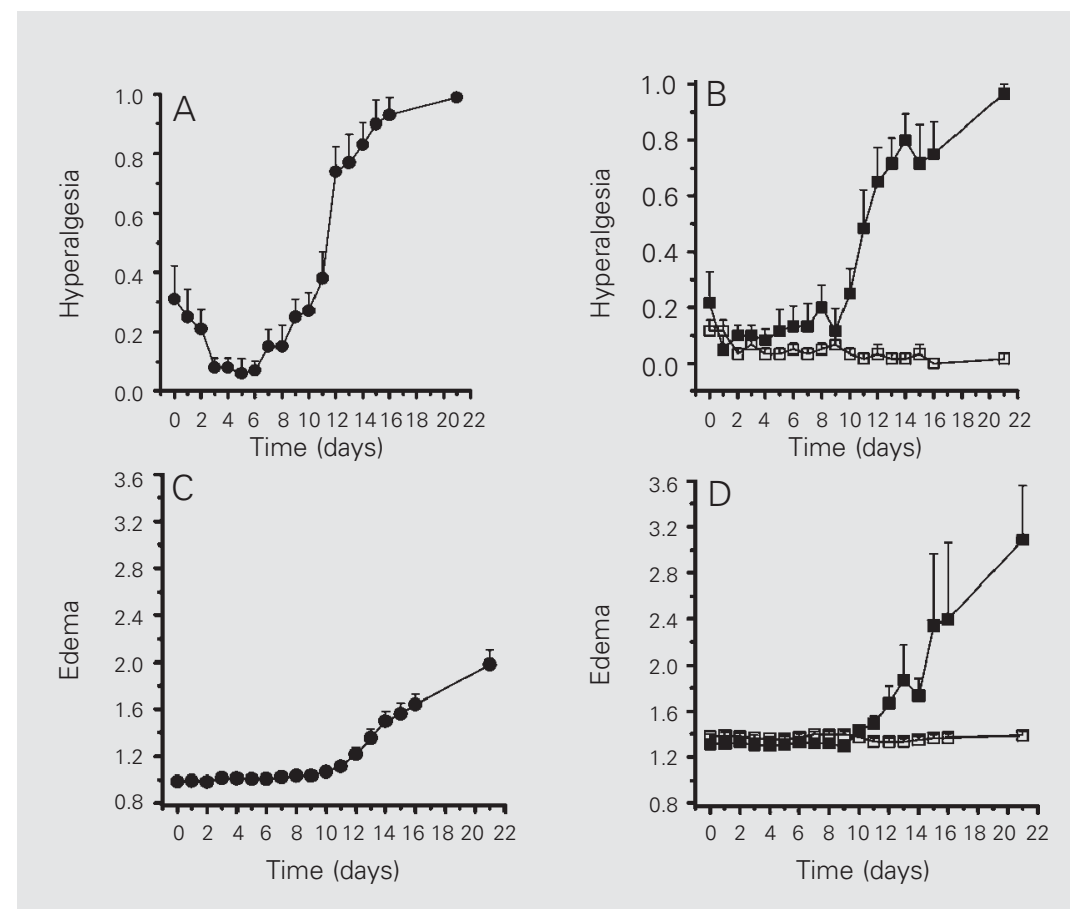

Figure 1. Development of hyperalgesia $(\mathrm{A}, \mathrm{B})$ and paw edema $(\mathrm{C}, \mathrm{D})$ following the induction of arthritis in juvenile ( $N=10$, circles), old responder ( $N=3$, open squares) and old nonresponder ( $N=9$, filled squares) rats. Arthritis was induced at day zero by subcutaneous injection of $0.2 \mathrm{ml}$ of an emulsion containing $400 \mu \mathrm{g}$ Mycobacterium butyricum into the base of the rat tail. Hyperalgesia $(A, B)$ is reported as the mean number of vocalizations ( \pm SEM) in response to 5 flexions of the tarsotibial joints assessed daily. Old responder animals (filled squares, 6 of 12) presented the same profile of hyperalgesia as juvenile animals. Data for nonresponder animals (open squares) are also shown. Edema (C, D) is reported as the mean increase in volume \pm SEM (in $\mathrm{ml}$ ) found for both hind paws, also assessed daily. Old responder rats presented more intense paw edema on the 21st day of observation compared with juvenile animals (compare $\mathrm{D}$ with $\mathrm{C}$ ) and more interindividual variation. 
The maximum percent increase in paw volume on the 21 st day was $100 \%$ for juvenile animals and $135 \%$ for old responder animals (Table 1). However, there was no significant difference in maximum edema values between groups. Differences from the initial paw volume values shown by old arthritic rats from zero to 9 days of induction prob-

Table 1. Effect of bilateral adrenalectomy on the increase in paw volume in rats with adjuvant-induced arthritis.

\begin{tabular}{lccc}
\hline Group & $\begin{array}{c}\text { Increase in } \\
\text { paw volume (\%) }\end{array}$ & $P_{1}$ & $P_{2}$ \\
\hline $\begin{array}{l}\text { Control 1 } \\
\text { Juvenile }(\mathrm{N}=10)\end{array}$ & $100 \pm 11.7$ & - & - \\
Control 2 & & & - \\
$\quad$ Old responder $(\mathrm{N}=3)$ & $0.7 \pm 1.99$ & $>0.05$ & - \\
$\quad$ Old nonresponder $(\mathrm{N}=9)$ & $31.2 \pm 15.2$ & & \\
Control 3 (sham operated) & $9.9 \pm 2.36$ & $<0.05$ & $<0.05$ \\
$\quad$ Old responder $(\mathrm{N}=4)$ & & & $<0.05$ \\
$\quad$ Old nonresponder $(\mathrm{N}=5)$ & $36.7 \pm 2.22$ & $<0.05$ & $>0.05$ \\
Bilateral adrenalectomy & & & \\
$\quad$ Old responder $(\mathrm{N}=7)$ & & &
\end{tabular}

Data are reported as the mean \pm SEM of the percent of hind paw volume increase compared to the contralateral paw on the 21 st day after the initiation of arthritis. $\mathrm{P}_{1}$ : data compared with data for the juvenile group; $P_{2}$ : data for old animals were compared with data for control 2 and data for bilaterally adrenalectomized old responders were compared with data for control 3 (sham-operated animals). Data were analyzed statistically by two-way ANOVA for multiple comparisons, with the level of significance set at $\mathrm{P}<0.05$.
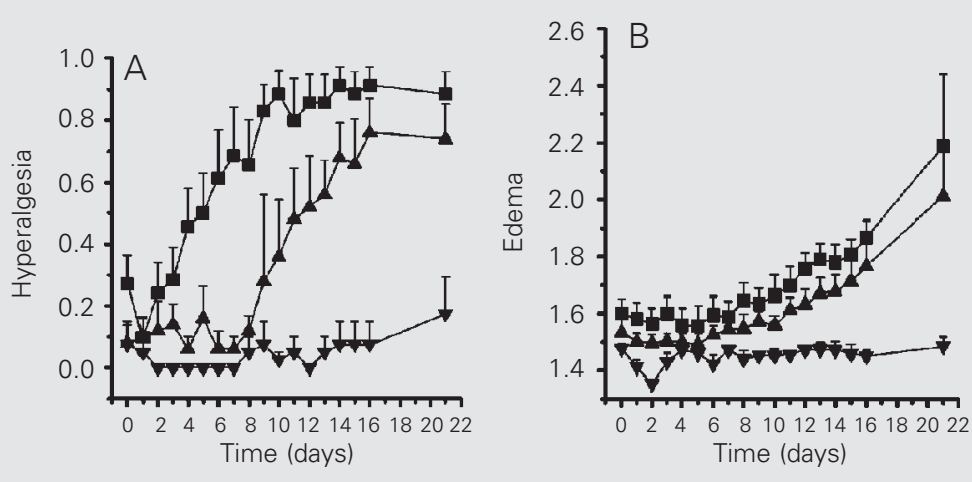

Figure 2. Effect of bilateral adrenalectomy ( $N=7$, squares) on the hyperalgesia ( $A$ ) and edema (B) responses of old arthritic rats (controls: $N=4$, triangles $=$ responders, $N=5$, inverted triangles $=$ nonresponders). Adrenalectomy or sham operation $(N=9)$ was performed 5 days before induction of arthritis (day zero). Animals were classified as nonresponders when the mean hyperalgesia and edema measurements were less than a 0.3 or $0.3 \mathrm{ml}$ increase, respectively. The induction of arthritis and the hyperalgesia and edema measurements were reported as described in the legend to Figure 1. ably reflected their higher initial weight in comparison to juvenile animals (Figure 1D). Moreover, at the end of the experiment on the 21st day of observation, joint deformities shown by responder old arthritic animals were less evident than in juvenile animals (data not shown).

\section{Effect of adrenal removal on the response of old animals to adjuvant arthritis}

Removal of both adrenal glands from old rats rendered all the animals hyperalgesic (filled squares), as illustrated in Figure 2A. The figure shows that animals submitted to surgery but without removal of the two glands, i.e., sham-operated (control) animals, could still be divided into responder (filled triangles) and nonresponder (inverted filled triangles) animals in relation to hyperalgesia measurements, as previously shown for old arthritic (non-operated) rats. In addition, adrenalectomy reduced the latency for hyperalgesia development from the 10th to 5th day following disease induction (Figure 2A). However, all adrenalectomized old responder rats presented paw edema of the same intensity as sham-operated old responder animals. Surprisingly, the intensity of this response in both groups of animals was significantly lower (a $\sim 30 \%$ increase) than that observed in non-operated old responder animals (135\%; Table 1) even though surgery had occurred 5 days before.

\section{Effect of dexamethasone administration} following bilateral adrenalectomy on the hyperalgesia and paw edema responses of old arthritic rats

Dexamethasone was administered immediately after surgery at two daily doses $(0.1$ $\mathrm{mg} / \mathrm{kg}$, open lozenges, or $0.01 \mathrm{mg} / \mathrm{kg}$, filled lozenges) to groups of bilaterally adrenalectomized animals to determine the role of corticosteroids in arthritis development in old rats. Chronic dexamethasone adminis- 
tration at both doses significantly inhibited hyperalgesia and paw edema development (Figure 3A,B) to the level of the old nonresponder animals, as shown in Figure 1B and $\mathrm{D}$, respectively. The reduction of hyperalgesia and paw edema from the level of control adrenalectomized old rats to the level of nonresponder animals was not observed in adrenalectomized old animals treated with 3 $\mathrm{mg} / \mathrm{kg}$ celecoxib (Figure 4A,B), except for the first three days of hyperalgesia response (Figure 4A). This dose of celecoxib significantly inhibited the hyperalgesia induced by a single injection of $250 \mu \mathrm{g}$ carrageenan into the rat paw (data not shown).

\section{Discussion}

The model of adjuvant-induced arthritis in rats is a useful tool to study the pathophysiology of rheumatoid arthritis, a chronic inflammatory reaction, especially because the experimental model and the human disease share various signs and symptoms (3). Furthermore, the activity profile of antiinflammatory drugs used for treatment of rheumatoid arthritis correlates well with the data obtained for the same drugs in the experimental rat model $(12,14)$. In the present study, while searching for an ideal size of rats for other purposes, we occasionally observed that older control animals presented a low frequency of arthritis (hyperalgesia and edema responses) when challenged. This prompted us to investigate the hind paw edema and hyperalgesia responses in adjuvant-challenged old animals in comparison with their "good" responder juvenile counterparts. We observed that, indeed, both arthritic hyperalgesia and paw edema responses were much less frequent in older animals, representing 50 and $25 \%$ of the frequencies found in juvenile rats, respectively. However, the ability of old responder animals to react to an imposed hyperalgesic stimulus was preserved since these animals showed a response of the same magnitude as that of young rats, with the two curves practically overlapping (Figure 1A and B). Since the hyperalgesic response to the adjuvant remained unchanged in old responder animals, we conclude that the mechanisms involved in the hyperalgesic response are not altered with increasing age. In addition, the inflammatory (edema) response of old rats responding to adjuvant arthritis was even more pronounced than in juvenile animals, although the difference was not statistically significant. However, this could be related to the

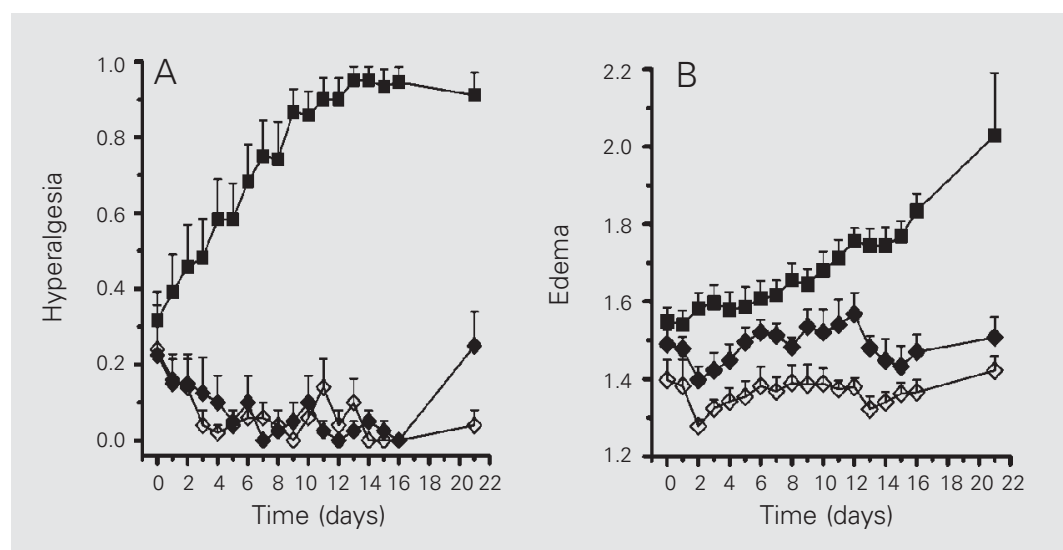

Figure 3. Effect of dexamethasone on the hyperalgesia (A) and edema (B) of adrenalectomized old arthritic rats (filled squares). Dexamethasone 0.1 (open lozenges) or $0.01 \mathrm{mg} / \mathrm{kg}$ (filled lozenges) was administered by gavage daily starting immediately after surgery, 5 days before induction of arthritis, and continued until the last day of the experiment. $\mathrm{N}=4$ 5/group. See legend to Figure 1 for a description of the induction of arthritis.

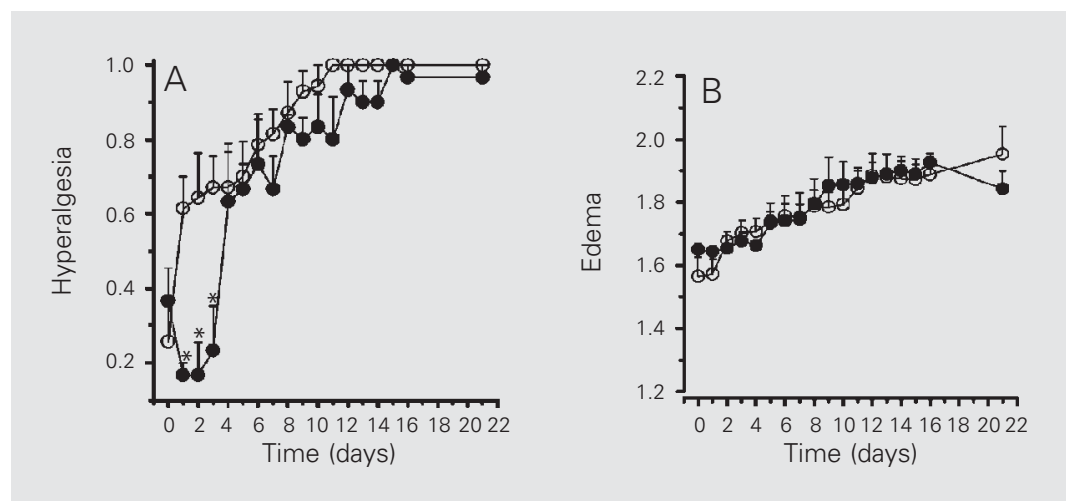

Figure 4. Effect of celecoxib (filled circles) on the hyperalgesia and edema responses of adrenalectomized old arthritic rats (control, open circles). Celecoxib (3 mg/kg) was administered daily by gavage starting immediately after surgery, 5 days before arthritis was induced, and continued until the last day of the experiment. $N=3 / g r o u p$. See legend to Figure 1 for a description of the induction of arthritis. ${ }^{*} \mathrm{P}<0.05$ compared to untreated adrenalectomized old arthritic rats (two-way ANOVA). 
fact that the paws of older rats, which are larger initially, can also expand more.

Since old rats present higher basal levels of circulating corticosteroids when submitted to stress (15), we determined whether removing the adrenals would affect the development of arthritis in old animals. In fact, an increase in the frequency of hyperalgesia and paw edema was observed in adrenalectomized old arthritic animals in relation to controls. In addition, the maximal increase in hyperalgesia in adrenalectomized old animals was attained earlier, around the 5th day, when compared with the hyperalgesia presented by juvenile rats (8th to 10th day). However, a different profile was observed in old animals in relation to the edema response following surgery. The edema response was of much lower intensity in operated animals (sham and adrenalectomized) than in nonoperated old animals (compare Figure 2 with Figure $1, \mathrm{P}<0.05$, two-way ANOVA). In addition, the responses were of the same (low) magnitude in sham- and bilaterally adrenalectomized animals compared to non-operated responder animals (a 30 vs 135\% increase, respectively). This suggests that the edema response was affected by either the surgery or anesthesia itself and was independent of the presence of adrenals in old animals.

Another important observation in the present study was the survival of adrenalectomized old arthritic rats. It has been reported that juvenile arthritic rats do not survive adrenalectomy (16). Our results suggest that the adrenal glands play a minor role in the survival of aging rats. However, the hyperalgesia responses were increased in terms of frequency and of earlier onset following bilateral adrenalectomy in relation to control animals, thus indicating that integrity of the adrenals is important at least for the modulation of the arthritic pain response in old animals.

We administered exogenous dexamethasone, a standard anti-inflammatory steroid (17), to old arthritic animals submitted to bilateral adrenalectomy to determine whether corticosteroids were the substances from the adrenal glands involved in such inhibitory response. As shown in Figure 3, corticotherapy administered to these challenged animals reversed the increase in the hyperalgesia previously seen in adrenalectomized animals. Dexamethasonetreated (old) animals showed flatter hyperalgesia and edema intensity curves comparable to the responses displayed by non-arthritic animals. The ability of $\mathrm{T}$ cells from old individuals and animals to respond to an immunological challenge is known to be reduced $(18,19)$. In fact, we have observed that rat mononuclear cells from old rats produce and release significantly lower amounts of hyperalgesic substances than mononuclear cells from young animals (Pereira LSM, unpublished observations). However, since inhibition of increased hyperalgesia and edema frequency after adrenalectomy was not consistently observed in old arthritic rats chronically treated with celecoxib, a specific cyclooxygenase inhibitor (20-22), we concluded that those responses were specifically modulated, at least in part, by corticosterone. A slight and shortlived reduction of the increased hyperalgesia frequency by celecoxib was observed in old arthritic rats submitted to adrenalectomy (Figure 4A), confirming studies which showed the participation of COX-2 in the early phase of arthritic pain (23). In the present study, this participation was shown in old rats. However, this chronic treatment with celecoxib did not affect the edema response of old arthritic rats at any time studied.

Our results show that old rats present a lower frequency of arthritic hyperalgesia and edema compared with their juvenile counterparts. Our data also pointed out a partial role for the adrenal glands, especially in terms of endogenous corticosteroids, in this modulation. In particular, inhibition of the hyperalgesia but not of the edema presented by old arthritic animals was observed with dexamethasone, an exogenous steroid, but not with celecoxib, a specific COX-2 inhibitor. 


\section{References}

1. Sewell KL \& Trentham DE (1993). Pathogenesis of rheumatoid arthritis. Lancet, 341: 283-286.

2. Meenan RF \& Yellin FH (1981). The impact of chronic disease: a sociomedical profile of rheumatoid arthritis. Arthritis and Rheumatism, 25: 544-554.

3. Pearson CM \& Wood FD (1963). Studies of arthritis and other lesions induced in rats by the injection of mycobacterial adjuvant. American Journal of Pathology, 42: 93-95.

4. Owen RT (1980). Adjuvant induced polyarthritis - an overview. Methods and Findings in Experimental and Clinical Pharmacology, 2: 199204.

5. Colpaert FC, Meert T, De Witthand P \& Schmitt P (1982). Further evidence validating adjuvant arthritis as an experimental model of chronic pain in the rat. Life Sciences, 31: 67-75.

6. Tatsuo MAKF, Carvalho WM, Silva CV, Miranda AEG, Ferreira SH \& Francischi JN (1994). Analgesic and antiinflammatory effects of dipyrone in rat adjuvant arthritis model. Inflammation, 18: 399-405.

7. Francischi JN, Pereira LSM \& Castro MS (1997). Cyclosporin inhibits hyperalgesia and edema in arthritic rats: role of the central nervous system. Brazilian Journal of Medical and Biological Research, 30: 101-111.

8. Francischi JN, Yokoro CM, Poole S, Tafuri LW, Cunha FQ \& Teixeira MM (2000). Anti-inflammatory and analgesic effects of the phosphodiesterase 4 inhibitor rolipram in a rat model of arthritis. European Journal of Pharmacology, 399: 243-249.

9. Dayan M, Segal R, Globerson A, Habut B, Shearer GM \& Mozes E (2000). Effect of aging on cytokine production in normal and experimental systemic lupus erythematosus-afflicted mice. Experimental Gerontology, 35: 225-236.

10. Hench PS, Kendall EC, Slocumb CH \& Polley HF (1949). The effect of a hormone of the adrenal cortex (17-hydroxy-11-dehydrocorticosterone: compound E) and of pituitary adrenocorticotropic hormone on rheumatoid arthritis. Proceedings of the Staff Meetings of the Mayo Clinic, 24: 181-197.

11. Zimmerman M (1983). Ethical guidelines for investigations of experimental pain in conscious animals. Pain, 16: 109-110.
12. Capetola JR, Shriver AD \& Rosenthale EM (1980). Suprofen, a new peripheral analgesic. Journal of Pharmacology and Experimental Therapeutics, 214: 16-23.

13. Zarrow MX, Yochim JM, McCarthy JL \& Sanborn RC (1964). Experimental Endocrinology. 1st edn. Academic Press, New York, NY, USA.

14. Jaffe BD, Kerr JS, Jones EA, Giannaras JV \& McGowan M (1989). The effect of immunomodulating drugs on adjuvant-induced arthritis in Lewis rats. Agents and Actions, 27: 345-346.

15. Sabatino F, Masoro EJ, McMahan CA \& Kuhn RW (1991). Assessment of the role of the glucocorticoid system in aging processes and in the action of food restriction. Journal of Gerontology, 46: B171B179.

16. Harbuz MS, Leonard JP, Lightman SL \& Cuzner ML (1993). HPA axis responses to acute stress and adrenalectomy during adjuvant-induced arthritis in the rat. American Journal of Physiology, 264: R179R185.

17. Chrousos GP (1995). The hypothalamic-pituitary-adrenal axis and immune-mediated inflammation. New England Journal of Medicine, 332: 1351-1362.

18. Li M, Walter R, Torres C \& Sierra F (2000). Impaired signal transduction in mitogen activated rat splenic lymphocytes during aging. Mechanisms of Ageing and Development, 113: 85-99.

19. Yung RL (2000). Changes in immune function with age. Rheumatic Disease Clinics of North America, 26: 455-473.

20. Seibert K, Zhang Y, Leahy K, Hauser S, Masferrer J, Perkins W, Lee $L$ \& Isakson P (1994). Pharmacological and biochemical demonstration of the role of cyclooxygenase 2 in inflammation and pain. Proceedings of the National Academy of Sciences, USA, 91: 1201312017.

21. Hawkett CJ (1999). COX-2 inhibitors. Lancet, 353: 307-314.

22. Vane JR, Bakhle YS \& Botting RM (1998). Cyclooxygenases 1 and 2. Annual Review of Pharmacology and Toxicology, 38: 97-120.

23. Vanegas H \& Schaible H-G (2001). Prostaglandins and cyclooxygenases in the spinal cord. Progress in Neurobiology, 64: 327-363. 